\title{
Meson fluctuations and thermodynamics of the Polyakov-loop-extended quark-meson model
}

\author{
V. Skokov, ${ }^{1, *}$ B. Stokić, ${ }^{1}$ B. Friman, ${ }^{1}$ and K. Redlich ${ }^{2,3}$ \\ ${ }^{1}$ GSI Helmholtzzentrum für Schwerionenforschung, D-64291 Darmstadt, Germany \\ ${ }^{2}$ Institute of Theoretical Physics, University of Wroclaw, PL-50204 Wroctaw, Poland \\ ${ }^{3}$ Theory Division, CERN, CH-1211 Geneva 23, Switzerland
}

(Received 16 April 2010; published 26 July 2010)

\begin{abstract}
Thermodynamics of the Polyakov-loop-extended two-flavor chiral quark-meson model (PQM) is explored. The analysis of the PQM model is based on the functional renormalization group method. An appropriate truncation of the effective action with quarks coupled to background gluonic fields is introduced. Within this scheme, we derive the renormalization group flow equation for the scale-dependent thermodynamic potential at finite temperature and density in the presence of a symmetry breaking external field. The influence of fluctuations and of the background gluon field on the properties of net-quark number density fluctuations and their higher moments is explored. We study the dependence of the kurtosis of quark-number fluctuations on the pion mass and show that, in the presence of a symmetry-breaking term, the fluctuations lead to a smoothing of observables near the crossover transition.
\end{abstract}

DOI: 10.1103/PhysRevC.82.015206

PACS number(s): 24.85.+p, 21.65.-f, 25.75.-q, 24.60.-k

\section{INTRODUCTION}

The phase diagram of strongly interacting matter at nonzero baryon density and high temperature has been a subject of growing interest in recent years. Calculations done within lattice gauge theory (LGT) show a clear separation between the confined, hadronic, and deconfined, quark-gluon plasma, phases at finite temperature [1]. Quantum chromodynamics (QCD) exhibits both dynamical chiral symmetry breaking and confinement at finite temperature and densities. However, because QCD thermodynamics at large baryon densities is still not accessible from first-principle LGT calculations, many phenomenological models and effective theories have been developed.

The hadronic properties at low energy as well as the nature of the chiral phase transition at finite temperature and densities have been successfully described and explored in such effective models. Also, the physics of color deconfinement and its relation to chiral symmetry breaking has been recently studied in terms of effective chiral models [2-13]. The idea to extend the existing chiral models, such as the Nambu-JonaLasinio (NJL) or the chiral quark-meson model, by introducing couplings of quarks to uniform temporal background gauge fields (Polyakov loops) was an important step forward in these studies [5,13].

It was shown that the Polyakov-loop-extended NambuJona-Lasinio (PNJL) [7] or Polyakov-loop-extended quarkmeson (PQM) [13] models reproduce essential properties of QCD thermodynamics obtained in the LGT calculations already in the mean-field approximation. However, to correctly account for the critical behavior and scaling properties near the chiral phase transitions, one needs to go beyond the mean-field approximation and include quantum fluctuations and nonperturbative dynamics. This can be accounted for by using the functional renormalization

\footnotetext{
*V.Skokov@gsi.de
}

group (FRG) [14-17]. Until now this method was applied in the NJL and quark-meson model, where the FRG equation was formulated for quarks coupled to meson fields [18-24].

In this article we propose a truncation of the PQM model which is suitable for a FRG analysis. We introduce an additional coupling of the chiral condensate to a gluonic background via Polyakov loops. In this way the Polyakov loop dynamics is represented by a corresponding background temporal gauge field. We thus use the FRG approach to include fluctuations of the meson fields, while the Polyakov loop is treated on a mean-field level. Consequently, our calculation lacks an FRG description of the fluctuations of the Polyakov loops. However, already in this approximation we find an important effect of the interactions of quarks with the effective gluon fields on the thermodynamics. For comparison we also present results for the chiral quark-meson (QM) model without Polyakov loops in the FRG approach to make the difference in the thermodynamics of the two effective models more transparent and to emphasize the role of the gluonic background field. We also compare with the PQM and QM models in the mean-field approximation.

In this study we use the Taylor expansion method to describe the thermodynamics at finite chemical potential $\mu$. We discuss the influence of fluctuations and the background gluon field on the properties of the net-quark number density susceptibilities. Results on the properties of generalized susceptibilities of the net quark number density in the presence of mesonic fluctuations in a gluonic background are presented. We also explore ways to reduce the dependence on the ultraviolet cutoff in the FRG approach.

The article is organized as follows: In Sec. II we introduce the PQM model. In Sec. III we formulate the renormalization group equation for the thermodynamic potential in the PQM model. In Sec. IV we consider the mean-field approximation. In Sec. $\mathrm{V}$ we discuss the critical properties and thermodynamics of the PQM model in the presence of mesonic fluctuations. 


\section{THE POLYAKOV-QUARK-MESON MODEL}

The chiral quark-meson model is used as an effective realization of the low-energy sector of the QCD. However, because the local $\mathrm{SU}\left(N_{c}\right)$ invariance of QCD is replaced with a global symmetry, one cannot describe deconfinement phenomena in this model. Recently, it was argued that, by connecting the chiral quark-meson model with the Polyakov loop potential, the confining properties of QCD can be approximately accounted for $[5,12,13]$. Consequently, the PQM model effectively combines both the chiral and the confining properties of QCD.

The Lagrangian of the PQM model reads

$$
\begin{aligned}
\mathcal{L}= & \bar{q}\left[i \not D-g\left(\sigma+i \gamma_{5} \vec{\tau} \vec{\pi}\right)\right] q+\frac{1}{2}\left(\partial_{\mu} \sigma\right)^{2}+\frac{1}{2}\left(\partial_{\mu} \vec{\pi}\right)^{2} \\
& -U(\sigma, \vec{\pi})-\mathcal{U}\left(\ell, \ell^{*}\right) .
\end{aligned}
$$

The coupling between the effective gluon field and the quarks is implemented through the covariant derivative

$$
D_{\mu}=\partial_{\mu}-i A_{\mu},
$$

where $A_{\mu}=g A_{\mu}^{a} \lambda^{a} / 2$. The spatial components of the gluon field are neglected; that is, $A_{\mu}=\delta_{\mu 0} A_{0}$. Moreover, $\mathcal{U}\left(\ell, \ell^{*}\right)$ is the effective potential for the gluon field expressed in terms of the thermal expectation values of the color trace of the Polyakov loop and its conjugate

$$
\ell=\frac{1}{N_{c}}\left\langle\operatorname{Tr}_{c} L(\vec{x})\right\rangle, \quad \ell^{*}=\frac{1}{N_{c}}\left\langle\operatorname{Tr}_{c} L^{\dagger}(\vec{x})\right\rangle,
$$

with

$$
L(\vec{x})=\mathcal{P} \exp \left[i \int_{0}^{\beta} d \tau A_{4}(\vec{x}, \tau)\right],
$$

where $\mathcal{P}$ stands for path ordering, $\beta=1 / T$, and $A_{4}=i A_{0}$.

The $O(4)$ representation of the meson fields is $\phi=$ $(\sigma, \vec{\pi})$ and the corresponding $\mathrm{SU}(2)_{L} \otimes \mathrm{SU}(2)_{R}$ chiral representation is given by $\sigma+i \vec{\tau} \cdot \vec{\pi} \gamma_{5}$. There are $N_{f}^{2}=4$ mesonic degrees of freedom coupled to $N_{f}=2$ flavors of quarks.

The purely mesonic potential of the model, $U(\sigma, \vec{\pi})$, is defined as

$$
U(\sigma, \vec{\pi})=\frac{\lambda}{4}\left(\sigma^{2}+\vec{\pi}^{2}-v^{2}\right)^{2}-c \sigma,
$$

while the effective potential of the gluon field is parametrized to preserve the $Z(3)$ invariance:

$$
\frac{\mathcal{U}\left(\ell, \ell^{*}\right)}{T^{4}}=-\frac{b_{2}(T)}{2} \ell^{*} \ell-\frac{b_{3}}{6}\left(\ell^{3}+\ell^{* 3}\right)+\frac{b_{4}}{4}\left(\ell^{*} \ell\right)^{2} .
$$

The parameters,

$$
b_{2}(T)=a_{0}+a_{1}\left(\frac{T_{0}}{T}\right)+a_{2}\left(\frac{T_{0}}{T}\right)^{2}+a_{3}\left(\frac{T_{0}}{T}\right)^{3},
$$

with $a_{0}=6.75, a_{1}=-1.95, a_{2}=2.625, a_{3}=-7.44, b_{3}=$ 0.75 , and $b_{4}=7.5$ were chosen to reproduce the equation of state of pure gauge degrees of freedom calculated on the lattice
[7]. At the temperature $T_{0}=270 \mathrm{MeV}$, the critical temperature of pure gauge theory, the potential (6) yields a first-order phase transition.

\section{THE FRG METHOD IN THE PQM MODEL}

The FRG is an important tool for addressing nonperturbative problems within quantum field theory. It is based on an infrared regularization of the full propagator, with the momentum scale parameter $k$. The regularization turns the corresponding effective action into a scaledependent functional $\Gamma_{k}$ [14-17]. The change of $\Gamma_{k}$ with the change of the momentum scale is described by the flow equation

$$
\begin{aligned}
\partial_{k} \Gamma_{k}[\Phi, \psi]= & \frac{1}{2} \operatorname{Tr}\left\{\partial_{k} R_{k B}\left(\Gamma_{k}^{(2,0)}[\Phi, \psi]+R_{k B}\right)^{-1}\right\} \\
& -\operatorname{Tr}\left\{\partial_{k} R_{k F}\left(\Gamma_{k}^{(0,2)}[\Phi, \psi]+R_{k F}\right)^{-1}\right\},
\end{aligned}
$$

where $\Gamma_{k}^{(2,0)}$ and $\Gamma_{k}^{(0,2)}$ denote the second functional derivative of $\Gamma_{k}[\Phi, \psi]$ with respect to the bosonic $(\Phi)$ and fermionic $(\psi)$ fields, respectively. These derivatives correspond to the inverse of the full propagators at the scale $k$. The trace in Eq. (8) denotes a momentum integration and a summation over all internal indices (e.g., flavor, color, and/or Dirac). Here $\Phi$ and $\psi$ denote bosonic and fermionic fields, respectively. The effective average action, $\Gamma_{k}$, governs the dynamics of a theory at a momentum scale $k$ and interpolates between the bare action, $\Gamma_{k=\Lambda} \equiv S$, and the full quantum effective action, $\Gamma_{k=0}=\Gamma$. The regulator $R_{k}$, which suppresses the small momentum modes, is to some extent arbitrary [17]. The derivative of this function, $\partial_{k} R_{k}$, implements the Wilsonian idea of successively integrating out momentum shells.

In the PQM model the formulation of the FRG flow equation (8) would require an implementation of the Polyakov loop as a dynamical field. However, at present it is not clear how this can be accomplished. Hence, in this exploratory calculation we treat the Polyakov loop on the mean-field level. This allows us to formulate the FRG flow equation for the truncated effective PQM action in Euclidean space-time $(t \rightarrow-i \tau)$ :

$$
\begin{aligned}
\Gamma_{k}= & \int d^{4} x\left\{\frac{1}{2}\left(\partial_{\mu} \phi\right)^{2}+\bar{q} \not \partial q\right. \\
& \left.+g \bar{q}\left(\sigma+i \vec{\tau} \cdot \vec{\pi} \gamma_{5}\right) q+U_{k}(\rho)\right\},
\end{aligned}
$$

where the field $\rho$ is given by

$$
\rho=\frac{1}{2} \phi^{2}=\frac{1}{2}\left(\sigma^{2}+\vec{\pi}^{2}\right) .
$$

The finite quark chemical potential, $\mu$, and the gauge potential, $A_{0}$, are introduced through the following substitution of the time derivative

$$
\partial_{\tau} \rightarrow \partial_{\tau}-\left(\mu+i A_{4}\right)
$$

Because the gauge potential, $A_{0}$, is treated as an effective background gluonic field, which does not flow with the scale parameter $k$, the explicit contribution of the Polyakov loop potential $\mathcal{U}$ to the truncated effective action is suppressed at this stage. Later on we restore $\mathcal{U}$. 
As in the previous studies [25], we employ the optimized regulator functions [26] in the numerical implementation. For bosons, this regulator depends only on the spatial components of the momentum,

$$
R_{B, k}^{\mathrm{opt}}\left(\mathbf{q}^{2}\right)=\left(k^{2}-\mathbf{q}^{2}\right) \theta\left(k^{2}-\mathbf{q}^{2}\right),
$$

whereas for fermions we use [25]

$$
R_{F, k}^{\mathrm{opt}}(\mathbf{q})=\left(\sqrt{\frac{\left(q_{0}+i \alpha_{0}\right)^{2}+k^{2}}{\left(q_{0}+i \alpha_{0}\right)^{2}+\mathbf{q}^{2}}}-1\right)\left(\not q+i \gamma^{0} \alpha_{0}\right) \theta\left(k^{2}-\mathbf{q}^{2}\right),
$$

where $\alpha_{0}=\mu+i A_{0}$ is modified owing to the coupling to the background gluon field.

Using Eq. (9), with the regulators (12)-(13), and the relation $\Omega=T \Gamma$ [27], we obtain the flow equation for the scale-dependent grand canonical potential for the quark and mesonic subsystems,

$$
\begin{aligned}
\partial_{k} \Omega_{k}\left(\ell, \ell^{*} ; T, \mu\right) \\
=\frac{k^{4}}{12 \pi^{2}}\left\{\frac{3}{E_{\pi}}\left[1+2 n_{B}\left(E_{\pi} ; T\right)\right]+\frac{1}{E_{\sigma}}\left[1+2 n_{B}\left(E_{\sigma} ; T\right)\right]\right. \\
\left.\quad-\frac{4 N_{c} N_{f}}{E_{q}}\left[1-N\left(\ell, \ell^{*} ; T, \mu\right)-\bar{N}\left(\ell, \ell^{*} ; T, \mu\right)\right]\right\} .
\end{aligned}
$$

Here $n_{B}\left(E_{\pi, \sigma} ; T\right)$ is the bosonic distribution function

$$
n_{B}\left(E_{\pi, \sigma} ; T\right)=\frac{1}{\exp \left(E_{\pi, \sigma} / T\right)-1},
$$

with the pion and $\sigma$ energy

$$
E_{\pi}=\sqrt{k^{2}+\bar{\Omega}_{k}^{\prime}}, \quad E_{\sigma}=\sqrt{k^{2}+\bar{\Omega}_{k}^{\prime}+2 \rho \bar{\Omega}_{k}^{\prime \prime}},
$$

where the primes denote derivatives with respect to $\rho$ and $\bar{\Omega}=\Omega+c \sigma$. The functions $N\left(\ell, \ell^{*} ; T, \mu\right)$ and $\bar{N}\left(\ell, \ell^{*} ; T, \mu\right)$, defined by

$$
\begin{aligned}
& N\left(\ell, \ell^{*} ; T, \mu\right)=\frac{1+2 \ell^{*} \exp \left[\beta\left(E_{q}-\mu\right)\right]+\ell \exp \left[2 \beta\left(E_{q}-\mu\right)\right]}{1+3 \ell \exp \left[2 \beta\left(E_{q}-\mu\right)\right]+3 \ell^{*} \exp \left[\beta\left(E_{q}-\mu\right)\right]+\exp \left[3 \beta\left(E_{q}-\mu\right)\right]}, \\
& \bar{N}\left(\ell, \ell^{*} ; T, \mu\right)=N\left(\ell^{*}, \ell ; T,-\mu\right)
\end{aligned}
$$

are fermionic distributions, modified due to the coupling to gluons, and

$$
E_{q}=\sqrt{k^{2}+2 g^{2} \rho}
$$

is the quark energy. In the absence of the background gluon field, for example, in the QM model, $\ell, \ell^{*} \rightarrow 1$ and the FermiDirac distribution function is recovered; $N(1,1 ; T, \mu)=$ $n_{F}\left(E_{q} ; T, \mu\right)=1 /\left\{\exp \left[\left(E_{q}-\mu\right) / T\right]+1\right\}$.

To solve the flow equation (14), we expand $\bar{\Omega}_{k}(T, \mu)$ in a Taylor series around the scale-dependent minimum at $\sigma_{k}=$ $\sqrt{2 \rho_{k}}$ :

$$
\bar{\Omega}_{k}=\sum_{i=0}^{m} \frac{a_{i, k}}{i !}\left(\rho-\rho_{k}\right)^{i} .
$$

As in Ref. [25], we truncate the expansion at $m=3$. The higher-order expansion terms are not expected to be important for the present study. The Taylor coefficients, $a_{i, k}$, are functions of the scale, $k$, the temperature, $T$, the chemical potential, $\mu$, and the Polyakov loop, $\ell$ and $\ell^{*}$.

The minimum of the thermodynamic potential is determined by the stationarity condition

$$
\left.\frac{d \Omega_{k}}{d \sigma}\right|_{\sigma=\sigma_{k}}=\left.\frac{d \bar{\Omega}_{k}}{d \sigma}\right|_{\sigma=\sigma_{k}}-c=0
$$

The set of flow equations for each expansion coefficient, $a_{i, k}$, arising from the Eq. (18) is solved numerically with the ultraviolet cutoff $\Lambda=1.2 \mathrm{GeV}$. The initial conditions, $a_{i, k=\Lambda}$ and $c$, are chosen to reproduce the physical pion mass $m_{\pi}=138 \mathrm{MeV}$, the pion decay constant $f_{\pi}=93 \mathrm{MeV}$, the $\sigma$ mass $m_{\sigma}=700 \mathrm{MeV}$ and the constituent quark mass $m_{q}=335 \mathrm{MeV}$ at the scale $k=0$ in vacuum. The symmetry breaking term, $c=m_{\pi}^{2} f_{\pi}$, corresponds to an external field and consequently does not flow. In this work, we neglect the flow of the Yukawa coupling, $g$, which is not expected to be significant (see, e.g., Refs. [18,28]).

By solving Eq. (18) we obtain a thermodynamic potential for the quark and mesonic subsystems, $\Omega_{k \rightarrow 0}\left(\ell, \ell^{*} ; T, \mu\right)$, as a function of Polyakov loop variables $\ell$ and $\ell^{*}$. So far these variables are arbitrary. The full thermodynamic potential in the PQM model, including quark, meson, and gluon degrees of freedom, is obtained by adding to $\Omega_{k \rightarrow 0}\left(\ell, \ell^{*} ; T, \mu\right)$ the effective gluon potential $\mathcal{U}\left(\ell, \ell^{*}\right)$ from Eq. (6),

$$
\Omega\left(\ell, \ell^{*} ; T, \mu\right)=\Omega_{k \rightarrow 0}\left(\ell, \ell^{*} ; T, \mu\right)+\mathcal{U}\left(\ell, \ell^{*}\right) .
$$

At a given $T$ and $\mu$, the Polyakov loop variables $\ell$ and $\ell^{*}$ are determined by the stationarity conditions:

$$
\begin{aligned}
\frac{\partial}{\partial \ell} \Omega\left(\ell, \ell^{*} ; T, \mu\right) & =0, \\
\frac{\partial}{\partial \ell^{*}} \Omega\left(\ell, \ell^{*} ; T, \mu\right) & =0 .
\end{aligned}
$$

Within the FRG approach, the thermodynamic potential of the QM model is obtained from Eq. (20), by dropping the effective Polyakov loop potential and setting $\ell=\ell^{*}=1$.

In the following we explore the thermodynamics of the PQM-FRG model. The role of the gluonic sector is assessed by juxtaposing the results obtained within the PQM and QM models, while the effect of mesonic fluctuations in the PQM model are deduced by comparing with the mean-field approximation. 


\section{THE MEAN-FIELD APPROXIMATION}

The importance of mesonic fluctuations is best illustrated by comparing the FRG approach with the mean-field (MF) approximation for mesons. In the latter, both quantum and thermal fluctuations are neglected and the mesonic fields are replaced by their classical expectation values. In the formulation presented in the previous section, the MF approximation is recovered by omitting the pion- and $\sigma$-meson contributions in Eq. (14). Alternatively, this approximation can be obtained directly from the partition function for the Lagrangian (1) by integrating out the quark degrees of freedom. These approaches are equivalent up to a surface term, which is negligible for large cutoffs, $\Lambda \rightarrow \infty$. We explicitly retain the divergent vacuum contribution, which is regularized by an ultraviolet cutoff $\Lambda_{\mathrm{MF}}$. The importance of this contribution was demonstrated in Refs. [29,30].

A detailed derivation of the MF approximation of PMQ model can be found in Ref. [13] and references therein. Here we present only the final result for thermodynamic potential

$$
\Omega_{\mathrm{MF}}=\mathcal{U}\left(\ell, \ell^{*}\right)+U(\langle\sigma\rangle,\langle\pi\rangle=0)+\Omega_{q \bar{q}}\left(\langle\sigma\rangle, \ell, \ell^{*}\right) .
$$

The contribution of quarks with mass $m_{q}=g\langle\sigma\rangle$ is given by

$$
\begin{aligned}
\Omega_{q \bar{q}}\left(\langle\sigma\rangle, \ell, \ell^{*}\right)= & -2 N_{f} T \int \frac{d^{3} p}{(2 \pi)^{3}}\left\{\frac{N_{c} E_{q}}{T} \theta\left(\Lambda_{\mathrm{MF}}^{2}-p^{2}\right)\right. \\
& +\ln g^{(+)}\left(\langle\sigma\rangle, \ell, \ell^{*} ; T, \mu\right) \\
& \left.+\ln g^{(-)}\left(\langle\sigma\rangle, \ell, \ell^{*} ; T, \mu\right)\right\}
\end{aligned}
$$

where

$$
\begin{aligned}
g^{(+)}\left(\langle\sigma\rangle, \ell, \ell^{*} ; T, \mu\right)= & 1+3 \ell \exp \left[-\left(E_{q}-\mu\right) / T\right] \\
& +3 \ell^{*} \exp \left[-2\left(E_{q}-\mu\right) / T\right] \\
& +\exp \left[-3\left(E_{q}-\mu\right) / T\right], \\
g^{(-)}\left(\langle\sigma\rangle, \ell, \ell^{*} ; T, \mu\right)= & g^{(+)}\left(\langle\sigma\rangle, \ell^{*}, \ell ; T,-\mu\right),
\end{aligned}
$$

and $E_{q}=\sqrt{p^{2}+m_{q}^{2}}$ is the quark quasiparticle energy. The equations of motion for the MFs are obtained by requiring that the thermodynamic potential be stationary with respect to changes of $\sigma, \ell$, and $\ell^{*}$ :

$$
\frac{\partial \Omega_{\mathrm{MF}}}{\partial \sigma}=\frac{\partial \Omega_{\mathrm{MF}}}{\partial \ell}=\frac{\partial \Omega_{\mathrm{MF}}}{\partial \ell^{*}}=0 .
$$

The model parameters are fixed to reproduce the same vacuum physics as in the FRG calculation, as described in the previous section. The additional free parameter, $\Lambda_{\mathrm{MF}}$, is chosen to reproduce the quark condensate in vacuum $\langle\bar{u} u\rangle=-(260 \mathrm{MeV})^{3}$; we find $\Lambda_{\mathrm{MF}}=674 \mathrm{MeV}$.

\section{FLUCTUATIONS AND THERMODYNAMICS OF THE PQM MODEL}

The thermodynamic potential obtained in Sec. III can be used to explore the influence of the gluonic background field on the thermodynamics in the PQM model including the effect of meson fluctuations. In previous studies of the
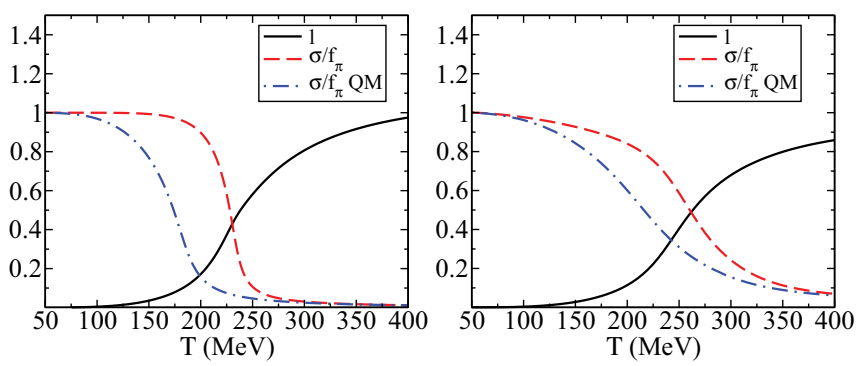

FIG. 1. (Color online) Thermal average of the Polyakov loop, $\ell$, and of the order parameter of the chiral phase transition, $\langle\sigma\rangle$, as functions of temperature at zero baryon chemical potential in the mean-field approximation (left panel) and in FRG approach (right panel). The solid and dashed lines are obtained in the PQM model, while the dash-dotted lines correspond to the QM model.

chiral quark-meson model within the FRG approach, it was shown that nonperturbative meson contributions modify the position of the chiral boundary and the critical end point (CEP) in the $(T, \mu)$ plane. The pseudocritical temperature and chemical potential are usually identified by a maximum in the temperature derivative of the order parameter or in the chiral susceptibility. In Figs. 1 and 2 we show the temperature dependence of $\langle\sigma\rangle$ and the Polyakov loop $\ell$ and of their temperature derivatives computed in the FRG approach, as well as in the MF approximation for the PQM and QM models.

As expected, for a physical pion mass the model exhibits a smooth crossover as a function of temperature. Moreover, as shown in Fig. 2, the temperature derivatives of both the Polyakov loop and the chiral order parameter exhibit peak structures at approximately the same temperature, $T_{c}=$ $257 \mathrm{MeV}$ in the PQM-FRG and $T_{c}=230 \mathrm{MeV}$ in the PQMMF model. In general, the relation between the deconfinement and chiral phase transitions is not well established. Recent LGT calculations lead to conflicting conclusions concerning the relative position of these transitions $[1,31]$.

A comparison of the QM and PQM models shows that the inclusion of gluon degrees of freedom shifts the chiral phase transition to higher temperatures both with and without mesonic fluctuations. The latter lead to a smoothing of the transition, as shown in Figs. 1 and 2.
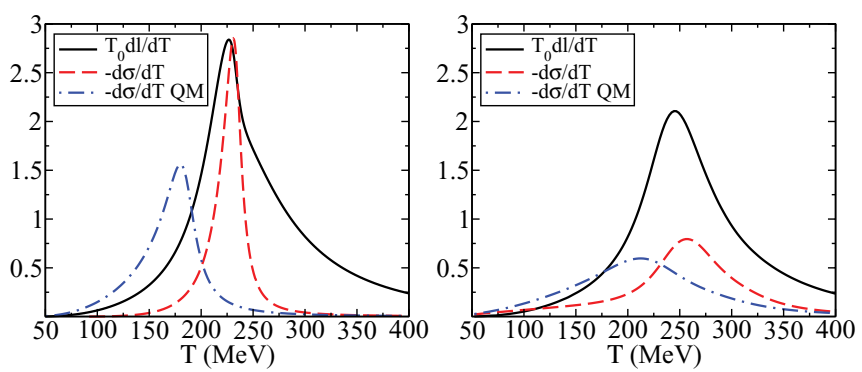

FIG. 2. (Color online) Temperature derivatives of the thermal average of the Polyakov loop, $\ell$, and of the order parameter of the chiral phase transition, $\langle\sigma\rangle$, as functions of temperature at zero baryon chemical potential in the mean-field approximation (left panel) and in the FRG approach (right panel). The notation is the same as in Fig. 1. 


\section{A. Quark-number fluctuations}

To explore the influence of nonzero baryon chemical potential on the thermodynamics, we employ the Taylor expansion in $\mu / T$ around $\mu=0$ applied in LGT [1] and in model calculations [32,33]. In particular, we obtain the expansion of the thermodynamic pressure,

$$
\frac{p(T, \mu)}{T^{4}}=\sum_{n=0}^{\infty} \frac{1}{n !} c_{n}(T)\left(\frac{\mu}{T}\right)^{n},
$$

where

$$
c_{n}(T)=\left.\frac{\partial^{n}\left[p(T, \mu) / T^{4}\right]}{\partial(\mu / T)^{n}}\right|_{\mu=0} .
$$

The expansion coefficients $c_{n}(T)$ are generalized susceptibilities that characterize the fluctuations of the net quark number $\delta N_{q}=N_{q}-\left\langle N_{q}\right\rangle$ at vanishing chemical potential [34-36]. In particular, the first two nonvanishing derivatives, $c_{2}$ and $c_{4}$, are the second- and fourth-order cumulants:

$$
\begin{aligned}
& c_{2}=\frac{\chi_{q}}{T^{2}}=\frac{1}{V T^{3}}\left\langle\left(\delta N_{q}\right)^{2}\right\rangle, \\
& c_{4}=\frac{1}{V T^{3}}\left\langle\left(\delta N_{q}\right)^{4}\right\rangle-3\left\langle\left(\delta N_{q}\right)^{2}\right\rangle^{2},
\end{aligned}
$$

where $\chi_{q}$ is the regular quark number susceptibility.

The temperature dependence of the coefficients $c_{2}$ and $c_{4}$, obtained in the MF approximation and in the FRG approach, is shown in Figs. 3 and 4 . The coefficient $c_{2}$ increases monotonously with temperature. In the MF approximation, $c_{2}$ increases rapidly in the critical region and approaches the ideal gas limit at high temperatures. The FRG results show a rather different behavior at high temperatures. The corresponding susceptibility is strongly suppressed above the chiral transition, owing to the ultraviolet cutoff. The contribution of momenta beyond the cutoff to the thermodynamics is missing. To obtain the correct high-temperature behavior of the susceptibilities and other observables, one may supplement the FRG with the perturbative contribution of the high-momentum states. A simple model for implementing this correction was proposed in Ref. [37], where the corresponding contribution $(k>\Lambda)$ to the flow is approximated by that of a noninteracting gas of quarks and gluons. The flow equation for the high-
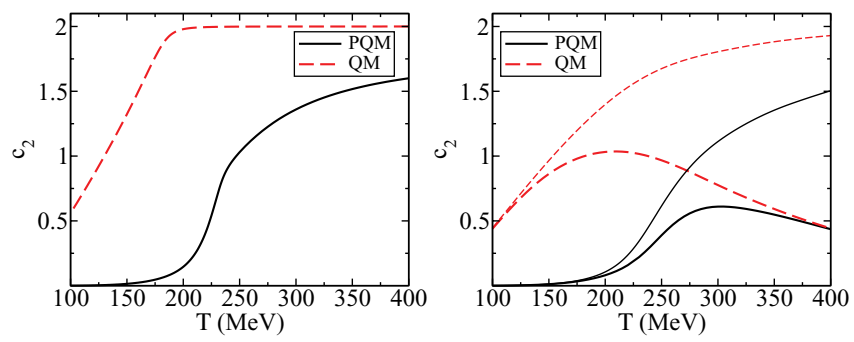

FIG. 3. (Color online) The coefficient $c_{2}$ as a function of temperature at zero baryon chemical potential for the PQM and QM models in the mean-field approximation (left panel) and in the FRG approach (right panel). The thin lines in the right panel indicate the FRG results after inclusion of the high-momentum flow (see text for details).
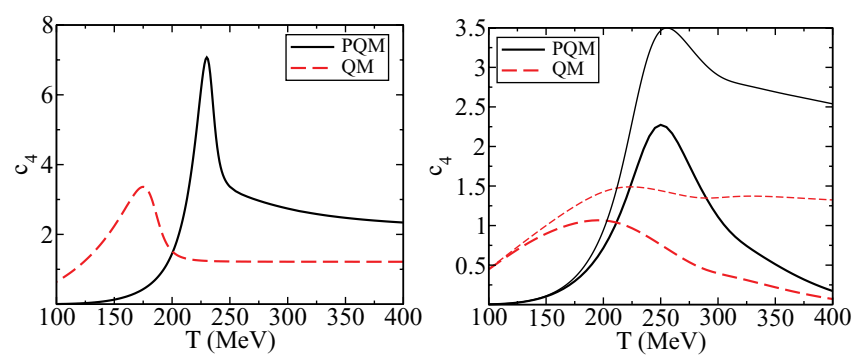

FIG. 4. (Color online) The coefficient $c_{4}$ as a function of temperature at zero baryon chemical potential for the PQM and QM models in the mean-field approximation (left panel) and in the FRG approach (right panel). As in Fig. 3, the thin lines indicate the FRG results after inclusion of the high-momentum flow (see text for details).

momentum contribution to the QM model then reads

$$
\begin{aligned}
\partial_{k} \Omega_{k}^{\Lambda}(T, \mu)= & \frac{k^{3}}{12 \pi^{2}}\left\{2\left(N_{c}^{2}-1\right)\left[1+2 n_{B}(k ; T)\right]\right. \\
& \left.-4 N_{c} N_{f}\left[1-n_{F}(k ; T, \mu)-n_{F}(k ; T,-\mu)\right]\right\},
\end{aligned}
$$

where the dynamical quark mass is neglected; that is, we set $E_{q}=k$. For the PQM model we generalize this procedure by including the interaction of quarks with the Polyakov loop

$$
\begin{aligned}
\partial_{k} \Omega_{k}^{\Lambda}(T, \mu)= & -\frac{N_{c} N_{f} k^{3}}{3 \pi^{2}} \\
& \times\left[1-N\left(\ell, \ell^{*} ; T, \mu\right)-\bar{N}\left(\ell, \ell^{*} ; T, \mu\right)\right] .
\end{aligned}
$$

Because the effective gluon potential $\mathcal{U}\left(\ell, \ell^{*}\right)$ is fitted to reproduce the Stefan Boltzmann limit at high temperatures, the explicit gluon contribution is omitted for consistency.

We thus proceed as follows: Equation (31) or Eq. (32) is integrated from $k=\infty$ to $k=\Lambda$, where we switch to the QM or PQM flow equation [Eq. (14)]. The divergent terms in the high-momentum flow Eqs. (31) and (32) are independent of mesonic and gluonic fields and of temperature and chemical potential. Consequently, they can be absorbed in an unobservable constant shift of the thermodynamic potential.

In the right panels of Figs. 3 and 4 we show the results for $c_{2}$ and $c_{4}$ in both the QM and PQM models. We find a smooth transition between the high- and low-energy regimes of the theory. In the QM model, both in the MF approximation and in the FRG approach, the Stefan Boltzmann limit, $c_{2}=2$ and $c_{4}=12 / \pi^{2}$, is reached already at temperatures $T \sim 2 T_{c}$. This is, however, not the case in the PQM model, where even at relatively high temperatures, the fluctuations show large deviations from their ideal gas values.

The different asymptotic behavior of the two models is clearly a consequence of the coupling of the quarks to the Polyakov loop. In the PQM model the ideal gas values of $c_{2}$ and $c_{4}$ are reached when $m_{q} / T \ll 1$ and $L \simeq 1$. The first condition is indeed satisfied already at $T \sim 2 T_{c}$, while the second one is not fulfilled even at very high temperatures. This is a direct consequence of the Polyakov loop potential in the PQM Lagrangian, which has been fitted to lattice results for 
pure SU(3) gauge theory, where $L$ approaches its asymptotic value very slowly.

Clearly, such a model is at best a crude approximation to QCD; the rather complex gluons dynamics is replaced by the Polyakov loop effective potential $\mathcal{U}$ and the interaction of quarks with the Polyakov loop. Other effects like the interaction of quarks with spacelike Wilson loops as well as a possible $\mu$ dependence of the parameters of $\mathcal{U}$ are neglected. This results in a rather slow convergence of $c_{2}$ and $c_{4}$ to their asymptotic values at a high temperature.

In contrast to $c_{2}$, the coefficient $c_{4}$ shows a nonmonotonic behavior near the chiral pseudocritical temperature. As shown in Fig. $4, c_{4}$ exhibits a pronounced peak at the pseudocritical temperature. The shape of the peak is changed considerably when mesonic fluctuations are included; its maximum value is reduced while the width is enhanced. We note that the peak value obtained in the MF approximation is strongly dependent on the cutoff $\Lambda_{\mathrm{MF}}$, used to regularize the divergent vacuum contribution. A smaller value of the cutoff yields a larger maximum value of $c_{4}$. The significance of the fermion vacuum contribution was recently emphasized in Refs. [29,30].

The general trends of the MF calculation can be inferred from Landau's theory of critical phenomena, where the thermodynamic potential is assumed to be a polynomial in the order parameter $\sigma$

$$
\Omega(T, \mu ; \sigma)=\Omega_{\mathrm{bg}}(T, \mu)+\frac{1}{2} a(T, \mu) \sigma^{2}+\frac{1}{4} b \sigma^{4}+c \sigma .
$$

Here $\Omega_{\mathrm{bg}}(T, \mu)$ is a background contribution, independent of $\sigma$, and $c$ is a symmetry-breaking term. The coefficient $a$ is generally assumed to be a linear function of the temperature, $a=A\left(T-T_{c}\right)$, where $T_{c}$ is the critical temperature of the second-order phase transition for $c=0$. An extension to small, nonzero values of the chemical potential is achieved by assuming

$$
a(T, \mu)=A\left(T-T_{c}\right)+B \mu^{2},
$$

where both coefficients $A$ and $B$ are positive. In general, the effective quartic coupling constant, $b>0$, also depends on temperature and chemical potential. However this dependence is irrelevant for $T \simeq T_{c}$ and $\mu \simeq 0$. In the chiral limit, $c=0$, the $c_{2}$ and $c_{4}$ coefficients are easily obtained from Eq. (33):

$$
\begin{aligned}
c_{2} & =c_{2}^{\mathrm{bg}}+\frac{A B}{b T} \frac{T-T_{c}}{T} \theta\left(T_{c}-T\right), \\
c_{4} & =c_{4}^{\mathrm{bg}}+\frac{6 B^{2}}{b} \theta\left(T_{c}-T\right) .
\end{aligned}
$$

Thus, $c_{2}$ is not differentiable at the critical point, while $c_{4}$ exhibits a discontinuity. The background parts $c_{2}^{\mathrm{bg}}$ and $c_{4}^{\mathrm{bg}}$ are smooth functions of temperature and do not change the critical behavior. For a finite pion mass $(c \neq 0)$, the transition is of the crossover type and the sharp structures in $c_{2}$ and $c_{4}$ are smoothed. Consequently, in the QM and PQM models, the peak structure appearing in $c_{4}$ is attributable to the decreasing quark mass and thus closely connected to the restoration of chiral symmetry. By contrast, in the resonance gas model, the peak in $c_{4}$ is due to the increase contribution of higher resonances.
In general, the singular part of thermodynamic potential is in chiral limit controlled by the critical exponents of the three-dimensional $\mathrm{O}(4)$ symmetric spin model. Consequently, at zero chemical potential:

$$
\begin{aligned}
\Omega^{\text {sing }} & \sim\left(T-T_{c}\right)^{2-\alpha}, \\
c_{2}^{\text {sing }} & \sim\left(T-T_{c}\right)^{1-\alpha}, \\
c_{4}^{\text {sing }} & \sim\left(T-T_{c}\right)^{-\alpha} .
\end{aligned}
$$

Critical fluctuations renormalize the exponent $\alpha$ from its MF value $\alpha_{\mathrm{MF}}=0$ to $\alpha=-0.21$. Consequently, fluctuations lead to a weakening of the singularity in the chiral limit. This is reflected in a smoothing of the temperature dependence for finite pion mass, as seen in Figs. 3 and 4. We note that in QCD the singularity in $c_{2}$ and $c_{4}$ may be obscured by resonances, which presumably dominate the thermodynamics of the lowtemperature phase.

A nonvanishing background gluon field leads to qualitative changes of the quark fluctuations. In the low-temperature phase, there is a strong suppression of the quark fluctuations owing to their coupling to the Polyakov loop (see Figs. 3 and 4). This is because single and double quark modes are suppressed when the expectation value of the Polyakov loop, $\ell$ and $\ell^{\star}$, is small [see Eqs. (14) and (15), as well as Eqs. (24) and (25)].

Lattice studies of QCD at finite temperature and density, as well as chiral model calculations, show that the ratio (kurtosis)

$$
R_{4,2}=\frac{c_{4}}{c_{2}}
$$

is a useful probe of the deconfinement and chiral phase transitions $[35,36,38]$. In the high- and low-temperature regimes, the kurtosis reflects the net quark content of the dominant baryon number carrying effective degrees of freedom [35,38].

In Fig. 5 we show the ratio $R_{4,2}$ as a function of temperature. Both in the FRG approach and in the MF approximation for the PQM model, the kurtosis drops from $R_{4,2} \simeq 9$ to $R_{4,2}<1$ in the transition region, as expected owing to the change in quark content of the baryon carrying effective degrees of freedom [35]. At low temperatures the effective three quark states dominate, while at high temperatures single quarks prevail. In the MF approximation (see the left panel of Fig. 5), the kurtosis exhibits a well-defined peak at the transition temperature. The height of the peak depends on the value of the pion mass $[38,39]$. This dependence is illustrated
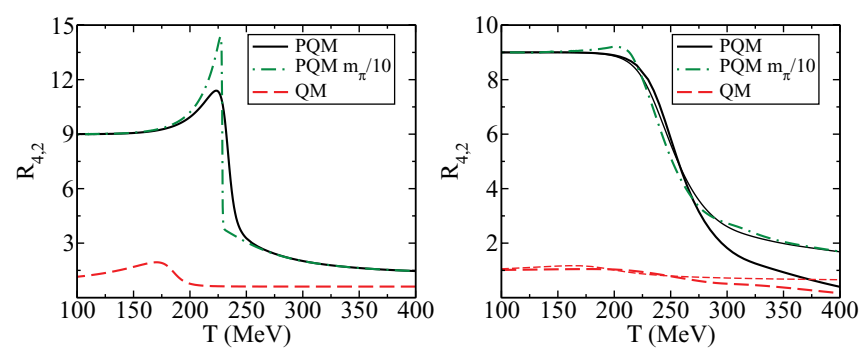

FIG. 5. (Color online) The kurtosis $R_{4,2}$ as a function of temperature at zero baryon chemical potential for the PQM and QM models in the mean-field approximation (left panel) and in the FRG approach (right panel). The thin lines indicate the FRG results after inclusion of the perturbative high-momentum modes (see text for details). 
in Fig. 5 both in the MF approximation and in the FRG approach. The inclusion of mesonic fluctuations weakens the dependence on the pion mass. Thus, for a physical value of $m_{\pi}$, the kurtosis decreases monotonously with temperature in the FRG approach. A peak in $R_{4,2}$ appears only for a pion mass below its physical value. In the MF approximation, the dependence on the pion mass is more pronounced, but still not as dramatic as found in Ref. [38]. This is attributable to the different treatments of the divergent fermion vacuum contribution. In Ref. [38] the vacuum term was subtracted at $T=0$ and its dependence on temperature was neglected. Here we regularize the vacuum term with an ultraviolet cutoff and retain its implicit temperature dependence through the in-medium quark mass. The role of the vacuum term in MF calculations and its influence on the chiral phase transition are explored in detail in Ref. [30].

In effective quark models where the Polyakov loop is neglected, the thermodynamics is governed by one-quark states at all temperatures. Hence, in the QM model the low-temperature limit of the kurtosis is $R_{4,2}=1$ rather than 9. Consequently, in such models, the interaction of quarks with the background Polyakov loop is essential for reproducing the low-temperature behavior of $R_{4,2}$ found in LGT calculations.

\section{SUMMARY AND CONCLUSIONS}

We have discussed thermodynamic properties of the PQM effective chiral model, including fluctuations within the FRG method. A truncation of the PQM model was introduced, which allowed us to extend previous renormalization group studies by introducing the coupling of fermions to the
Polyakov loop. We have thus formulated and solved the flow equation for the scale-dependent thermodynamic potential at finite temperature and density in the presence of a background gluonic field.

In our studies of the thermodynamics we have discussed the role of fluctuations for the critical properties of the model. The fluctuations of the net quark number density and the ratio of the fourth- to second-order cumulants, the kurtosis, were analyzed.

Furthermore, a comparison of the FRG approach with and without a gluon background field was performed. The influence of ultraviolet cutoff effects on the thermodynamics within the FRG approach was also discussed. We have shown that the FRG extended quark-meson model preserves the basic properties of the kurtosis obtained in LGT calculations only if the Polyakov loop is included in the flow equation. Thus, the extension of the FRG method proposed here, accounting for the coupling of fermions to background gluon fields, is of particular relevance for effective descriptions of QCD thermodynamics near the phase transition in terms of the quark-meson model.

\section{ACKNOWLEDGMENTS}

We acknowledge fruitful discussions with E. Nakano and B.-J. Schaefer. V.S. acknowledges stimulating discussions with K. Fukushima and support from the Frankfurt Institute for Advanced Studies (FIAS). K.R. acknowledges partial support from the Polish Ministry of Science (MEN) and the Alexander von Humboldt Foundation (AvH). B.S. gratefully acknowledges financial support from the Helmholtz Research School on Quark Matter Studies.
[1] M. Cheng et al., Phys. Rev. D 77, 014511 (2008).

[2] A. Gocksch and M. Ogilvie, Phys. Rev. D 31, 877 (1985).

[3] M. Buballa, Phys. Rep. 407, 205 (2005).

[4] P. N. Meisinger and M. C. Ogilvie, Phys. Lett. B 379, 163 (1996); P. N. Meisinger, T. R. Miller, and M. C. Ogilvie, Phys. Rev. D 65, 034009 (2002).

[5] K. Fukushima, Phys. Lett. B 591, 277 (2004).

[6] F. Sannino, Phys. Rev. D 66, 034013 (2002); A. Mocsy, F. Sannino, and K. Tuominen, Phys. Rev. Lett. 92, 182302 (2004).

[7] C. Ratti, M. A. Thaler, and W. Weise, Phys. Rev. D 73, 014019 (2006).

[8] C. Sasaki, B. Friman, and K. Redlich, Phys. Rev. D 77, 034024 (2008); Phys. Rev. Lett. 99, 232301 (2007); Phys. Rev. D 75, 074013 (2007).

[9] S. Digal, E. Laermann, and H. Satz, Eur. Phys. J. C 18, 583 (2001).

[10] E. Megias, E. R. Arriola, and L. L. Salcedo, Phys. Rev. D 74, 065005 (2006).

[11] E. M. Ilgenfritz and J. Kripfganz, Z. Phys. C 29, 79 (1985).

[12] K. Fukushima, Phys. Lett. B 553, 38 (2003); Phys. Rev. D 68, 045004 (2003).

[13] B.-J. Schaefer, J. M. Pawlowski, and J. Wambach, Phys. Rev. D 76, 074023 (2007).

[14] C. Wetterich, Phys. Lett. B 301, 90 (1993).
[15] T. R. Morris, Int. J. Mod. Phys. A 9, 2411 (1994).

[16] U. Ellwanger, Z. Phys. C 62, 503 (1994).

[17] J. Berges, N. Tetradis, and C. Wetterich, Phys. Rep. 363, 223 (2002).

[18] D. U. Jungnickel and C. Wetterich, Phys. Rev. D 53, 5142 (1996).

[19] J. Berges, D. U. Jungnickel, and C. Wetterich, Eur. Phys. J. C 13, 323 (2000)

[20] B.-J. Schaefer and J. Wambach, Nucl. Phys. A 757, 479 (2005).

[21] J. Berges, D. U. Jungnickel, and C. Wetterich, Phys. Rev. D 59, 034010 (1999).

[22] N. Tetradis, Nucl. Phys. A 726, 93 (2003).

[23] B.-J. Schaefer and H. J. Pirner, Nucl. Phys. A 660, 439 (1999).

[24] B.-J. Schaefer and J. Wambach, Phys. Rev. D 75, 085015 (2007).

[25] B. Stokic, B. Friman, and K. Redlich, Eur. Phys. J. C 67, 425 (2010).

[26] D. F. Litim, Phys. Rev. D 64, 105007 (2001).

[27] C. Wetterich, Phys. Rev. B 75, 085102 (2007).

[28] L. F. Palhares and E. S. Fraga, Phys. Rev. D 78, 025013 (2008); E. S. Fraga, L. F. Palhares, and M. B. Pinto, ibid. 79, 065026 (2009).

[29] E. Nakano, B. J. Schaefer, B. Stokic, B. Friman, and K. Redlich, Phys. Lett. B 682, 401 (2010).

[30] V. Skokov, B. Friman, E. Nakano, K. Redlich, and B. J. Schaefer, arXiv:1005.3166 [hep-ph]. 
[31] Y. Aoki, Z. Fodor, S. D. Katz, and K. K. Szabo, Phys. Lett. B 643, 46 (2006).

[32] C. Ratti, S. Roessner, M. A. Thaler, and W. Weise, Eur. Phys. J. C 49, 213 (2007).

[33] S. K. Ghosh, T. K. Mukherjee, M. G. Mustafa, and R. Ray, Phys. Rev. D 73, 114007 (2006).

[34] C. R. Allton, S. Ejiri, S. J. Hands, O. Kaczmarek, F. Karsch, E. Laermann, and C. Schmidt, Phys. Rev. D 68, 014507 (2003).

[35] S. Ejiri, F. Karsch, and K. Redlich, Phys. Lett. B 633, 275 (2006).
[36] F. Karsch, S. Ejiri, and K. Redlich, Nucl. Phys. A 774, 619 (2006); S. Ejiri et al., ibid. 774, 837 (2006).

[37] J. Braun, H. J. Pirner, and K. Schwenzer, Phys. Rev. D 70, 085016 (2004).

[38] B. Stokic, B. Friman, and K. Redlich, Phys. Lett. B 673, 192 (2009).

[39] F. Karsch, Proc. Sci. CPOD07, 026 (2007); C. Schmidt et al., J. Phys. G 35, 104093 (2008); F. Karsch, The QCD Critical Point, talk given at the INT Conference (INT-08-2b) (2008). 